Louisiana State University

LSU Digital Commons

Faculty Publications

Department of Mathematics

$1-1-2009$

\title{
Stabilization in a Two-Species Chemostat With Monod Growth Functions
}

Frédéric Mazenc

Michael Malisoff

Louisiana State University

Jérôme Harmand

Laboratoire de Biotechnologie de l'Environnement (LBE)

Follow this and additional works at: https://digitalcommons.Isu.edu/mathematics_pubs

\section{Recommended Citation}

Mazenc, F., Malisoff, M., \& Harmand, J. (2009). Stabilization in a Two-Species Chemostat With Monod Growth Functions. IEEE Transactions on Automatic Control, 54 (4), 855-861. https://doi.org/10.1109/ TAC.2008.2010964

This Article is brought to you for free and open access by the Department of Mathematics at LSU Digital Commons. It has been accepted for inclusion in Faculty Publications by an authorized administrator of LSU Digital Commons. For more information, please contact ir@lsu.edu. 


\title{
Stabilization in a Two-Species Chemostat with Monod Growth Functions ${ }^{1}$
}

\author{
Frédéric Mazenc, Michael Malisoff, and Jérôme Harmand ${ }^{2}$
}

\begin{abstract}
We design feedback controllers for two species chemostats so that an equilibrium with arbitrary prescribed species concentrations becomes globally asymptotically stable. Our design uses a new global, explicit, strict Lyapunov function construction, which allows us to quantify the effects of disturbances using the input-to-state stability paradigm. We assume that only a linear combination of the species concentrations is available for measurement. We illustrate our approach using a numerical example.
\end{abstract}

Key Words: Bioreactors, global stabilization, uncertain states, integral input-to-state stability

\section{INTRODUCTION}

This work continues our search (begun in [10], [11]) for ways to stabilize prescribed equilibrium behaviors in chemostats. See [2], [4], [12] for the fundamental role of chemostats in bioengineering. The basic model for a (well mixed) chemostat with two competing species is

$$
\dot{s}=D\left(s_{\text {in }}-s\right)-\frac{\mu_{1}(s)}{Y_{1}} X_{1}-\frac{\mu_{2}(s)}{Y_{2}} X_{2}, \quad \dot{X}_{i}=\left[\mu_{i}(s)-D\right] X_{i}, \quad i=1,2,
$$

evolving on $\mathcal{X}:=(0, \infty)^{3}\left[12\right.$, Chapter 1]. Here $s(t)$ is the concentration of the substrate; $X_{1}(t)$ and $X_{2}(t)$ are the concentrations of the two species of organism; the dilution rate $D(\cdot)$ and the input nutrient concentration $s_{\text {in }}(\cdot)$ are controllers that we will specify; $\mu_{1}$ and $\mu_{2}$ are given uptake functions; and $Y_{1}$ and $Y_{2}$ are positive constants called yield coefficients. We assume throughout that the $\mu_{i}$ s have the Monod form

$$
\mu_{i}(s)=\frac{K_{i} s}{L_{i}+s}
$$

\footnotetext{
1 Supported by NSF Grant 0424011. The authors thank Patrick De Leenheer for extensive and illuminating comments.

${ }^{2}$ Corresponding Author: M. Malisoff [Tel: (225) 578-1665. Fax: (225) 578-4276.] F. Mazenc is with Projet MERE INRIAINRA, UMR Analyse des Systèmes et Biométrie INRA, 2, pl. Viala, 34060 Montpellier, France, mazenc@supagro.inra.fr. M. Malisoff is with the Department of Mathematics, Louisiana State University, Baton Rouge, LA 70803-4918, malisoff@lsu.edu. J. Harmand is with Institut National de la Recherche Agronomique (INRA), UR050, Laboratoire de Biotechnologie de l'Environnement, Narbonne, F-11100, France, harmand@supagro.inra.fr.
} 
where $K_{i}$ and $L_{i}$ are positive constants we indicate below. Since $D$ is the ratio of the volumetric flow rate (with units of volume over time) to the constant reactor volume, it is proportional to the speed of the pump that supplies the reactor with fresh medium containing the nutrient.

The competitive exclusion principle implies that in classical chemostats with one limiting substrate and constant $D$ and $s_{\text {in }}$, at most one species can survive generically [12]. This is at odds with the observation that in real ecological systems, it is common for many species to coexist in equilibrium on one limiting nutrient. This paradox has motivated a great deal of research [4], [6]. Also, the species concentrations may not be available for measurement, and there may be actuator errors caused e.g. by variability in the speed of the pump supplying the fresh nutrient. Hence, it is important to quantify the robustness of any stabilizer to uncertainty.

This work addresses all of these issues by designing $s_{\text {in }}$ and $D(\cdot)$, depending only on a linear combination $Y=X_{1}+A X_{2}$ of the species concentrations (where $A$ is a given positive constant), that globally stabilize an equilibrium with arbitrary positive prescribed species concentrations. The design of stabilizing feedbacks depending only on a (weighted) sum of the species concentrations is well-motivated by applications where photometric methods preclude the possibility of separately measuring the individual species levels [4]. Another important feature is that our new construction for an explicit, multi-species chemostat, strict Lyapunov function makes it possible to quantify the effects of disturbances using integral input-to-state stability (iISS) and input-tostate stability (ISS) [13]; see [3], [14] for the essential role of (i)ISS in nonlinear control and applications. Our explicit Lyapunov function is a significant and original theoretical development.

\section{Assumptions And StABiLity Theorem}

Given $\mu_{i}$ 's as in (2), set $\chi(s)=\mu_{2}(s)-\mu_{1}(s)$. We always assume:

Assumption 1: There is a constant $s_{\star}>0$ such that (i) $\chi\left(s_{\star}\right)=0$, (ii) $\chi(s)<0$ when $0<s<s_{\star}$, and (iii) $\chi(s)>0$ when $s>s_{\star}$. In particular, $\mu_{1}\left(s_{\star}\right)=\mu_{2}\left(s_{\star}\right)$.

Simple calculations and our Assumption 1 readily yield

$$
\mu_{i}(s)-\mu_{i}(r)=\frac{L_{i}}{L_{i}+r} \frac{\mu_{i}(s)}{s}(s-r) \quad \forall s, r>0 \quad \text { for } i=1,2,
$$

$s_{\star}=\frac{L_{1} K_{2}-L_{2} K_{1}}{K_{1}-K_{2}}, \mu_{1}\left(s_{\star}\right)=\frac{L_{1} K_{2}-L_{2} K_{1}}{L_{1}-L_{2}}, K_{2}>K_{1}, K_{1} L_{2}>K_{2} L_{1}$, and $L_{2}>L_{1}$. We transform (1) with the output $Y=X_{1}+A X_{2}$, using $x_{1}=X_{1} / Y_{1}$ and $x_{2}=X_{2} / Y_{2}$, to get

$$
\dot{s}=D\left(s_{\text {in }}-s\right)-\mu_{1}(s) x_{1}-\mu_{2}(s) x_{2}, \quad \dot{x}_{i}=\left[\mu_{i}(s)-D\right] x_{i}, \quad i=1,2
$$


evolving on $\mathcal{X}=(0, \infty)^{3}$ (where $s_{\text {in }}$ and $D$ are to be chosen) and the output $y=Y / Y_{1}$ satisfying

$$
y=x_{1}+a x_{2}, \quad \text { where } a=A Y_{2} / Y_{1} .
$$

We always assume that $a \neq 1$. Given arbitrary prescribed $x_{1 \star}>0$ and $x_{2 \star}>0$, we set

$$
\begin{gathered}
s_{\text {in }}=s_{\star}+x_{1 \star}+x_{2 \star} ; \quad \tilde{x}_{i}=x_{i}-x_{i \star} ; \quad \tilde{s}=s-s_{\star} ; \\
\tilde{\Sigma}=\ln \left(\tilde{s}+s_{\star}\right)-\ln \left(s_{\star}\right) ; \text { and } \tilde{\xi}_{i}=\ln \left(\tilde{x}_{i}+x_{i \star}\right)-\ln \left(x_{i \star}\right)
\end{gathered}
$$

for $i=1,2$. For each trajectory $\left(s, x_{1}, x_{2}\right)(t)$ of $(4)$, we call the vector $\left(\tilde{\Sigma}, \tilde{\xi}_{1}, \tilde{\xi}_{2}\right)(t)$ as defined by (7) the (corresponding transformed) error vector. This transformation is essential for what follows because it produces a system evolving on $\mathbb{R}^{3}$. Also, $\left|\xi_{i}(t)\right| \rightarrow+\infty$ when $x_{i}(t) \rightarrow 0^{+}$ for each $i$, so the $\xi_{i}$ 's have the additional benefit of warning us when either species approaches extinction. We use the standard classes of comparison function $\mathcal{K}_{\infty}$ and $\mathcal{K} \mathcal{L}$; see [14]. We use the constants

$$
\begin{aligned}
& D_{\star}=\mu_{1}\left(s_{\star}\right), \quad \omega_{1}=\frac{D_{\star}}{20|a-1|}, \quad \omega_{2}=\frac{D_{\star}}{4 s_{\star}\left|\left(\frac{a}{L_{1}}-\frac{1}{L_{2}}\right)\right|+1}, \\
& \omega_{3}=\frac{D_{\star} s_{\star}}{20\left|(a-1) s_{\mathrm{in}}+s_{\star}^{2}\left(\frac{a}{L_{1}}-\frac{1}{L_{2}}\right)\right|+1}, \text { and } \omega_{4}=\frac{0.72 D_{\star} s_{\star}^{2}\left(\frac{1}{L_{1}}-\frac{1}{L_{2}}\right)}{\left[(a-1) s_{\mathrm{in}}+s_{\star}^{2}\left(\frac{a}{L_{1}}-\frac{1}{L_{2}}\right)\right]^{2}+1} .
\end{aligned}
$$

The following implies that $\left(s(t), x_{1}(t), x_{2}(t)\right) \rightarrow\left(s_{\star}, x_{1 \star}, x_{2 \star}\right)$ as $t \rightarrow+\infty$ for all trajectories of (4) (i.e., for all initial values in $\mathcal{X}$ ) hence globally asymptotically stable coexistence. It uses $\sigma(r)=\frac{r}{\sqrt{1+r^{2}}}$ but can be generalized to cover more general saturating functions.

Theorem 1: Consider the system (4) with output (5) with $a \neq 1$. Let $x_{1 \star}, x_{2 \star}>0$ be given, let $s_{\star}>0$ satisfy the requirements of Assumption 1, let $s_{\text {in }}$ satisfy (6), and let $\varepsilon \in$ $\left(0, \min \left\{\omega_{1}, \omega_{2}, \omega_{3}, \omega_{4}\right\}\right]$. Then $\left(s_{\star}, x_{1 \star}, x_{2 \star}\right)$ is a globally asymptotically stable equilibrium of (4) when $D(y)=D_{\star}-\varepsilon(a-1) \sigma\left(y-x_{1 \star}-a x_{2 \star}\right)$. More precisely, there exists $\beta \in \mathcal{K} \mathcal{L}$ such that for all trajectories $\left(s, x_{1}, x_{2}\right)(t)$ of (4) in closed loop with $s_{\text {in }}$ and $D$, the corresponding transformed error vector $\left(\tilde{\Sigma}, \tilde{\xi}_{1}, \tilde{\xi}_{2}\right)(t)$ from (7) satisfies $\left|\left(\tilde{\Sigma}, \tilde{\xi}_{1}, \tilde{\xi}_{2}\right)(t)\right| \leq \beta\left(\left|\left(\tilde{\Sigma}, \tilde{\xi}_{1}, \tilde{\xi}_{2}\right)(0)\right|, t\right)$ for all $t \geq 0$.

Remark 1: The controller $D$ from Theorem 1 yields coexistence because $x_{1 \star}>0$ and $x_{2 \star}>0$. Our motivation for this choice of $D$ is to stay as close as possible to the usual formulation in applications with constant $D$. However, we cannot pick $\varepsilon=0$ since that would make $D$ and $s_{\text {in }}$ constants, and so yield competitive exclusion [4]. Using standard stability arguments, it is possible to show that reducing $\varepsilon$ reduces the rate of convergence; we omit the proof of this 
fact due to space considerations. To make our analysis clear and easy to follow, we explain our choices of the $\omega_{i}$ 's in the course of the proof of Theorem 1 in Section V.

Remark 2: Throughout the next sections, we use more variables than needed. Indeed, doing that greatly simplifies the notations. Moreover, all (in)equalities should be understood to hold globally unless otherwise indicated, and we omit the arguments of our functions whenever this would not lead to confusion.

\section{RoBUSTNESS TO DistURBANCES}

Consider the perturbed chemostat error dynamics

$$
\left\{\begin{aligned}
\dot{\tilde{s}} & =\left[D(y)+\mathbf{d}_{2}\right]\left(s_{\mathrm{in}}+\mathbf{d}_{1}-s\right)-\mu_{1}(s) x_{1}-\mu_{2}(s) x_{2} \\
\dot{\tilde{x}}_{i} & =\left[\mu_{i}(s)-D(y)-\mathbf{d}_{2}\right] x_{i}, \quad i=1,2
\end{aligned}\right.
$$

with output (5) for $a \neq 1$, where $\tilde{s}=s-s_{\star} ; \tilde{x}_{i}=x_{i}-x_{i \star}$ for $i=1,2 ; s_{\text {in }}$ and $D$ are from Theorem 1; the $\mu_{i} \mathrm{~s}$ are as before (so Assumption 1 holds); and the unknown measurable disturbance $\mathbf{d}(t)=\left(\mathbf{d}_{1}(t), \mathbf{d}_{2}(t)\right)$ is bounded in sup norm $|\cdot|_{\infty}$ by a constant that we specify below. Let $\delta \mathcal{B}_{2}$ denote the closed ball of radius $\delta>0$ centered at $0 \in \mathbb{R}^{2}$. For each $\rho>0$, let $\mathcal{D}_{2}(\rho)$ denote the set of all measurable essentially bounded functions $\mathbf{d}:[0, \infty) \rightarrow \rho \mathcal{B}_{2}$. Fix arbitrary $x_{1 \star}>0$ and $x_{2 \star}>0$, and let $\varepsilon>0$ be as in the statement of Theorem 1. To simplify the notation, we set

$$
\begin{aligned}
& \bar{\alpha}=\frac{L_{1}+s_{\star}}{L_{1}}, \quad \bar{\beta}=\frac{x_{2 \star}}{x_{1 \star}} \frac{L_{2}+s_{\star}}{L_{2}}, A_{0}(\tilde{s})=\tilde{s}-s_{\star} \ln \left(1+\frac{\tilde{s}}{s_{\star}}\right)=s_{\star}\left(e^{\tilde{\Sigma}}-1-\tilde{\Sigma}\right), \\
& \text { and } A_{i}\left(\tilde{\xi}_{i}\right)=e^{\tilde{\xi}_{i}}-1-\tilde{\xi}_{i}=\frac{\tilde{x}_{i}}{x_{i \star}}-\ln \left(1+\frac{\tilde{x}_{i}}{x_{i \star}}\right)
\end{aligned}
$$

for $i=1$ and 2, where we used (7).

We show that (9) is iISS [14] in the variable $\left(\tilde{\Sigma}, \tilde{\xi}_{1}, \tilde{\xi}_{2}\right)$ (from (7) above) for disturbances $\mathbf{d} \in \mathcal{D}_{2}(\bar{\Delta})$ when $\bar{\Delta}$ is defined in (12), in the sense that there exist $\alpha \in \mathcal{K}_{\infty}$ and $\beta \in \mathcal{K} \mathcal{L}$ so that for all $\mathbf{d} \in \mathcal{D}_{2}(\bar{\Delta})$ and all corresponding closed-loop trajectories $\left(\tilde{s}(t), \tilde{x}_{1}(t), \tilde{x}_{2}(t)\right)$ of (9) for our controllers $D$ and $s_{\text {in }}$ (for all initial values), the corresponding transformed error vector $\left(\tilde{\Sigma}(t), \tilde{\xi}_{1}(t), \tilde{\xi}_{2}(t)\right)$ satisfies

$$
\alpha\left(\left|\left(\tilde{\Sigma}, \tilde{\xi}_{1}, \tilde{\xi}_{2}\right)(t)\right|\right) \leq \beta\left(\left|\left(\tilde{\Sigma}, \tilde{\xi}_{1}, \tilde{\xi}_{2}\right)(0)\right|, t\right)+\int_{0}^{t}|\mathbf{d}(r)| \mathrm{d} r \quad \forall t \geq 0 .
$$

We also show that (9) is ISS in $\left(\tilde{\Sigma}, \tilde{\xi}_{1}, \tilde{\xi}_{2}\right)$ when $\mathbf{d} \in \mathcal{D}_{2}\left(\bar{\Delta}^{b}\right)$ and $\bar{\Delta}^{b}$ is in (34), meaning there are $\beta \in \mathcal{K} \mathcal{L}$ and $\gamma \in \mathcal{K}_{\infty}$ so that for all $\mathbf{d} \in \mathcal{D}_{2}\left(\bar{\Delta}^{b}\right)$ and all closed-loop trajectories $\left(\tilde{s}, \tilde{x}_{1}, \tilde{x}_{2}\right)(t)$ 
of (9) for our $D$ and $s_{\text {in }}$ (for all initial values), $\left(\tilde{\Sigma}, \tilde{\xi}_{1}, \tilde{\xi}_{2}\right)(t)$ satisfies

$$
\left|\left(\tilde{\Sigma}, \tilde{\xi}_{1}, \tilde{\xi}_{2}\right)(t)\right| \leq \beta\left(\left|\left(\tilde{\Sigma}, \tilde{\xi}_{1}, \tilde{\xi}_{2}\right)(0)\right|, t\right)+\gamma\left(|\mathbf{d}|_{\infty}\right) \forall t \geq 0
$$

Notice that ISS guarantees persistence, since if for example we had $x_{2}(t) \rightarrow 0$ as $t \rightarrow t_{\star}^{-}$for some $t_{\star}>0$, then $\tilde{\xi}_{2}(t)=\ln \left(x_{2}(t)\right)-\ln \left(x_{2 \star}\right) \rightarrow-\infty$ as $t \rightarrow t_{\star}^{-}$, which would violate ISS. Similarly, iISS implies persistence when $\mathbf{d}$ is integrable.

To specify the bounds on $\mathbf{d}$, we introduce the constants

$$
\begin{aligned}
& C_{1}=\frac{s_{\star}}{10}\left(\frac{1}{L_{1}}-\frac{1}{L_{2}}\right) \varepsilon, \quad C_{2}=\frac{20}{19 D_{\star}}\left[\frac{\varepsilon^{2}}{4 C_{1}}\left(\frac{L_{1}+s_{\star}}{L_{1}} a-\frac{L_{2}+s_{\star}}{L_{2}}\right)^{2}+1\right], \\
& \kappa_{1}=\min \left\{1, \frac{D_{\star}}{5}\right\}+x_{1 \star}+x_{2 \star}+D_{\star}+\varepsilon|a-1|, \\
& \text { and } \kappa_{2}=\frac{\kappa_{1}}{2 s_{\mathrm{in}}^{2}}+2 C_{2}+\frac{1}{s_{\mathrm{in}}}\left(\kappa_{1} C_{2}+\max \left\{\bar{\alpha}, \frac{x_{1 \star}}{x_{2 \star}} \bar{\beta}\right\}\right) .
\end{aligned}
$$

See Section VII below for examples where the bounds on $\mathbf{d}$ are computed explicitly. Our iISS result is as follows:

Theorem 2: Under the preceding assumptions with

$$
\bar{\Delta}=\min \left\{1, \frac{2 D_{\star} s_{\star}}{5\left(3 \kappa_{1}+2 s_{\star}\right)}, \frac{1}{2 \kappa_{2}}\right\}
$$

(9) in closed loop with the controls provided by Theorem 1 is iISS in $\left(\tilde{\Sigma}, \tilde{\xi}_{1}, \tilde{\xi}_{2}\right)$ for disturbances $\mathbf{d} \in \mathcal{D}_{2}(\bar{\Delta})$.

See also Remarks 3-5 for ISS and related results under less stringent disturbance bounds.

\section{COMPARISON WITH KNOWN RESUlTS}

Lyapunov functions have not frequently been used to study stability in multi-species chemostats, and where they are used they are often nonstrict Lyapunov functions which do not lend themselves to robustness analysis. An exception is the one from [8]; see also [12, Theorem 4.1] and [7]. Strict Lyapunov functions for two species chemostats were constructed in [11], but no robustness to disturbances was established there. However, [11] provides simple linear feedback stabilizers that yield local asymptotic stability of a prescribed periodic trajectory. See [9], [15] where weak Lyapunov functions are used with variants of the LaSalle Invariance Principle.

This raises the important question of whether strict Lyapunov functions can be explicitly constructed for multi-species chemostats that are globally stabilized through static output feedbacks, and whether Lyapunov functions can be used to quantify the effects of uncertainty. For chemostats with one species that are made oscillating through a suitable $D(\cdot)$, this problem was 
solved in [10]. Our work owes a great deal to [4], [5], which stabilize chemostats in which only the sum of the species concentrations can be measured, using an appropriate $D$. However, [4] and [5] do not include our work since [4] does not rely on a Lyapunov approach and the Lyapunov functions from [5] are nonstrict and so do not lend themselves to ISS. See [6] for results where only the substrate level (multiplied by an a priori bounded error) is available for measurement.

\section{Proof of THEOREM 1}

Since $D_{\star}=\mu_{1}\left(s_{\star}\right)=\mu_{2}\left(s_{\star}\right)$, the change of feedback $D=D_{\star}+v$, (3) with the choice $r=s_{\star}$, and (7) transform (4) into

$$
\dot{\tilde{s}}=\left(D_{\star}+v\right)\left(s_{\text {in }}-s\right)-\mu_{1}(s) x_{1}-\mu_{2}(s) x_{2}, \quad \dot{\tilde{\xi}}_{i}=\frac{L_{i}}{L_{i}+s_{\star}} \frac{\mu_{i}(s)}{s} \tilde{s}-v, \quad i=1,2 .
$$

Step 1: Construction of a weak Lyapunov function

We first show that in terms of (10),

$$
V\left(\tilde{s}, \tilde{\xi}_{1}, \tilde{\xi}_{2}\right)=\frac{1}{x_{1 \star}} A_{0}(\tilde{s})+\bar{\alpha} A_{1}\left(\tilde{\xi}_{1}\right)+\bar{\beta} A_{2}\left(\tilde{\xi}_{2}\right)
$$

is a weak Lyapunov function for (13) in the sense that

$$
\dot{V}=-H(\tilde{s}) \tilde{s}^{2}+\frac{1}{x_{1 \star}} \frac{\tilde{s}}{s}\left(s_{\text {in }}-s\right) v-\left[\bar{\alpha} \frac{\tilde{x}_{1}}{x_{1 \star}}+\bar{\beta} \frac{\tilde{x}_{2}}{x_{2 \star}}\right] v
$$

holds along all trajectories of (13), where $H$ is defined by

$$
H(\tilde{s})=\frac{1}{s x_{1 \star}}\left[D_{\star}+\frac{\mu_{1}(s)-\mu_{1}\left(s_{\star}\right)}{\tilde{s}} x_{1 \star}+\frac{\mu_{2}(s)-\mu_{2}\left(s_{\star}\right)}{\tilde{s}} x_{2 \star}\right], \quad \tilde{s} \neq 0
$$

is everywhere positive. To this end, first notice that $\dot{A}_{0}=\frac{\tilde{s}}{s} \dot{\tilde{s}}, \dot{A}_{1}=\frac{\tilde{x}_{1}}{x_{1 \star}} \dot{\tilde{\xi}}_{1}$, and $\dot{A}_{2}=\frac{\tilde{x}_{2}}{x_{2 \star}} \dot{\dot{\xi}_{2}}$, by (7). Hence, along the trajectories of (13), we easily get

$$
\begin{aligned}
\dot{V}= & \frac{1}{x_{1 \star}} \frac{\tilde{s}}{s}\left(D_{\star}+v\right)\left(s_{\text {in }}-s\right)-\left[\bar{\alpha} \frac{\tilde{x}_{1}}{x_{1 \star}}+\bar{\beta} \frac{\tilde{x}_{2}}{x_{2 \star}}\right] v \\
& +\left[-x_{1}+\bar{\alpha} \frac{L_{1}}{L_{1}+s_{\star}} \tilde{x}_{1}\right] \frac{1}{x_{1 \star}} \frac{\mu_{1}(s)}{s} \tilde{s}+\left[-x_{2}+\bar{\beta} \frac{x_{1 \star}}{x_{2 \star}} \frac{L_{2}}{L_{2}+s_{\star}} \tilde{x}_{2}\right] \frac{1}{x_{1 \star}} \frac{\mu_{2}(s)}{s} \tilde{s} .
\end{aligned}
$$

Since $-x_{i}+\tilde{x}_{i}=-x_{i \star}$ for $i=1,2$, our choices of $\bar{\alpha}$ and $\bar{\beta}$ from (10) give

$$
\dot{V}=\frac{1}{x_{1 \star}}\left[D_{\star}\left(s_{\text {in }}-s\right)-x_{1 \star} \mu_{1}(s)-x_{2 \star} \mu_{2}(s)\right] \frac{\tilde{s}}{s}+\frac{1}{x_{1 \star}} \frac{\tilde{s}}{s}\left(s_{\text {in }}-s\right) v-\left[\bar{\alpha} \frac{\tilde{x}_{1}}{x_{1 \star}}+\bar{\beta} \frac{\tilde{x}_{2}}{x_{2 \star}}\right] v \text {. }
$$

The formulas $s_{\text {in }}=s_{\star}+x_{1 \star}+x_{2 \star}$ and $D_{\star}=\mu_{1}\left(s_{\star}\right)=\mu_{2}\left(s_{\star}\right)$ now easily give (15). 
Step 2: Construction of a strict Lyapunov function

Set $\tilde{y}=\tilde{x}_{1}+a \tilde{x}_{2}, \tilde{Z}=\tilde{s}+\tilde{x}_{1}+\tilde{x}_{2}, \Psi(r)=\frac{1}{2} r^{2}$, and

$$
V_{2}\left(\tilde{s}, \tilde{\xi}_{1}, \tilde{\xi}_{2}\right)=x_{1 \star} V\left(\tilde{s}, \tilde{\xi}_{1}, \tilde{\xi}_{2}\right)+\frac{20}{19 D_{\star}}\left[\frac{5 \varepsilon}{2 s_{\star}\left(\frac{1}{L_{1}}-\frac{1}{L_{2}}\right)}\left(\frac{L_{1}+s_{\star}}{L_{1}} a-\frac{L_{2}+s_{\star}}{L_{2}}\right)^{2}+1\right] \Psi(\tilde{Z})
$$

where $V$ is from (14). We show that $V_{2}$ is a strict Lyapunov function for (13) in the sense that its time derivative along the trajectories of (13) with $v=-\varepsilon(a-1) \sigma(\tilde{y})$ satisfies

$$
\dot{V}_{2} \leq-W_{2}, \quad \text { where } W_{2}\left(\tilde{s}, \tilde{\xi}_{1}, \tilde{\xi}_{2}\right)=\frac{D_{\star}}{4 s} \tilde{s}^{2}+\frac{s_{\star}}{10}\left[\frac{1}{L_{1}}-\frac{1}{L_{2}}\right] \varepsilon \tilde{y} \sigma(\tilde{y})+\tilde{Z}^{2}
$$

and $\tilde{x}_{i}$ and $\tilde{\xi}_{i}$ are related by (7). The fact that $W_{2}$ is positive definite follows from the facts that $a \neq 1, L_{2}>L_{1}$, and $r \mapsto r \sigma(r)$ is positive definite.

Since $\varepsilon \leq \omega_{1}$, our choice of $D$ gives $D(y)=D_{\star}+v \geq D_{\star}-\varepsilon(a-1) \geq \frac{19}{20} D_{\star}$ everywhere. Therefore, (4) gives

$$
\dot{\tilde{Z}}=D(y)\left[s_{\text {in }}-s-x_{1}-x_{2}\right]=-D(y) \tilde{Z}, \text { hence } \frac{d}{d t} \Psi(\tilde{Z}) \leq-\frac{19}{20} D_{\star} \tilde{Z}^{2} .
$$

Moreover, our assumption that $a \neq 1$ gives $\tilde{x}_{1}=(a[\tilde{Z}-\tilde{s}]-\tilde{y}) /(a-1)$ and $\tilde{x}_{2}=(\tilde{y}-\tilde{Z}+$ $\tilde{s}) /(a-1)$. Substituting the preceding formulas for the $\tilde{x}_{i}$ 's and (10) into (15), we easily get

$$
\begin{aligned}
\dot{V}= & -H(\tilde{s}) \tilde{s}^{2}+\frac{1}{x_{1 \star}}\left[\frac{1}{s} s_{\text {in }}+\frac{1}{a-1}\left(\frac{s_{\star}}{L_{1}} a-\frac{s_{\star}}{L_{2}}\right)\right] \tilde{s} v \\
& +\frac{s_{\star}}{x_{1 \star}(a-1)}\left[\frac{1}{L_{1}}-\frac{1}{L_{2}}\right] \tilde{y} v-\frac{1}{x_{1 \star}(a-1)}\left[\frac{L_{1}+s_{\star}}{L_{1}} a-\frac{L_{2}+s_{\star}}{L_{2}}\right] \tilde{Z} v .
\end{aligned}
$$

Taking $v=-\varepsilon(a-1) \sigma(\tilde{y})$ and recalling (16) gives

$$
\begin{aligned}
x_{1 \star} \dot{V} \leq & -\frac{D_{\star}}{s} \tilde{s}^{2}-\left[\frac{(a-1) s_{\text {in }}}{s}+\frac{\tilde{s}+s_{\star}}{s}\left(\frac{s_{\star}}{L_{1}} a-\frac{s_{\star}}{L_{2}}\right)\right] \tilde{s} \varepsilon \sigma(\tilde{y}) \\
& -s_{\star}\left[\frac{1}{L_{1}}-\frac{1}{L_{2}}\right] \varepsilon \tilde{y} \sigma(\tilde{y})+\left[\frac{L_{1}+s_{\star}}{L_{1}} a-\frac{L_{2}+s_{\star}}{L_{2}}\right] \tilde{Z} \varepsilon \sigma(\tilde{y}) .
\end{aligned}
$$

Since $\varepsilon \leq \omega_{2}$ and $|\sigma(\cdot)|$ is bounded by $1, \frac{\tilde{s}^{2}}{s}\left(\frac{s_{\star}}{L_{1}} a-\frac{s_{\star}}{L_{2}}\right) \varepsilon \sigma(\tilde{y}) \leq \frac{D_{\star}}{4 s} \tilde{s}^{2}$. Moreover,

$$
\left[\frac{L_{1}+s_{\star}}{L_{1}} a-\frac{L_{2}+s_{\star}}{L_{2}}\right] \tilde{Z} \varepsilon \sigma(\tilde{y}) \leq \frac{s_{\star}\left(\frac{1}{L_{1}}-\frac{1}{L_{2}}\right)}{10} \varepsilon \sigma(\tilde{y})^{2}+\frac{5 \varepsilon\left[\left(\frac{L_{1}+s_{\star}}{L_{1}} a-\frac{L_{2}+s_{\star}}{L_{2}}\right) \tilde{Z}\right]^{2}}{2 s_{\star}\left(\frac{1}{L_{1}}-\frac{1}{L_{2}}\right)} .
$$

Since $\sigma^{2}(\tilde{y}) \leq \tilde{y} \sigma(\tilde{y})$, we easily deduce that

$$
\begin{aligned}
x_{1 \star} \dot{V} \leq & -\frac{3 D_{\star}}{4 s} \tilde{s}^{2}-\frac{9 s_{\star}}{10}\left[\frac{1}{L_{1}}-\frac{1}{L_{2}}\right] \varepsilon \tilde{y} \sigma(\tilde{y})-\left[(a-1) s_{\text {in }}+s_{\star}^{2}\left(\frac{a}{L_{1}}-\frac{1}{L_{2}}\right)\right] \frac{\tilde{s}}{s} \varepsilon \sigma(\tilde{y}) \\
& +\frac{5 \varepsilon}{2 s_{\star}\left(\frac{1}{L_{1}}-\frac{1}{L_{2}}\right)}\left[\left(\frac{L_{1}+s_{\star}}{L_{1}} a-\frac{L_{2}+s_{\star}}{L_{2}}\right) \tilde{Z}\right]^{2} .
\end{aligned}
$$

It follows from (20) and our formula (18) for $V_{2}$ that

$$
\dot{V}_{2} \leq-\frac{3 D_{\star}}{4 s} \tilde{s}^{2}-\frac{9 s_{\star}}{10}\left[\frac{1}{L_{1}}-\frac{1}{L_{2}}\right] \varepsilon \tilde{y} \sigma(\tilde{y})-\tilde{Z}^{2}-\left[(a-1) s_{\text {in }}+s_{\star}^{2}\left(\frac{a}{L_{1}}-\frac{1}{L_{2}}\right)\right] \frac{\tilde{s}}{s} \varepsilon \sigma(\tilde{y}) .
$$


Notice that

$$
\left|\left\{\frac{\tilde{s}}{\sqrt{s}} \sqrt{D_{\star}}\right\}\left\{\frac{(a-1) s_{\text {in }}+s_{\star}^{2}\left(\frac{a}{L_{1}}-\frac{1}{L_{2}}\right)}{\sqrt{D_{\star} s}} \varepsilon \sigma(\tilde{y})\right\}\right| \leq \frac{D_{\star}}{4 s} \tilde{s}^{2}+\frac{\left[(a-1) s_{\text {in }}+s_{\star}^{2}\left(\frac{a}{L_{1}}-\frac{1}{L_{2}}\right)\right]^{2} \varepsilon^{2}}{D_{\star} s} \sigma^{2}(\tilde{y})
$$

for all $s>0$. Combining (21) and (22), it follows that

$$
\dot{V}_{2} \leq-\frac{D_{\star}}{2 s} \tilde{s}^{2}-\frac{9 s_{\star}}{10}\left[\frac{1}{L_{1}}-\frac{1}{L_{2}}\right] \varepsilon \tilde{y} \sigma(\tilde{y})-\tilde{Z}^{2}+\left\{\frac{\left[(a-1) s_{\mathrm{in}}+s_{\star}^{2}\left(\frac{a}{L_{1}}-\frac{1}{L_{2}}\right)\right]^{2} \varepsilon^{2}}{D_{\star} s} \sigma^{2}(\tilde{y})\right\} .
$$

We consider two cases. Case A: If $s \geq \frac{9}{10} s_{\star}$, then (23) and the fact that $\varepsilon \leq \omega_{4}$ give

$$
\dot{V}_{2} \leq-\frac{D_{\star}}{2 s} \tilde{s}^{2}-\frac{s_{\star}}{10}\left[\frac{1}{L_{1}}-\frac{1}{L_{2}}\right] \varepsilon \tilde{y} \sigma(\tilde{y})-\tilde{Z}^{2}
$$

because in this case, the term in braces in (23) is at most $\frac{10}{9}\left(\frac{1}{L_{1}}-\frac{1}{L_{2}}\right) s_{\star}(0.72) \varepsilon \sigma^{2}(\tilde{y}) \leq 0.8 s_{\star}\left(\frac{1}{L_{1}}-\right.$ $\left.\frac{1}{L_{2}}\right) \varepsilon \tilde{y} \sigma(\tilde{y})$. (Notice our use of 0.72 from (8).)

Case B: If $s \leq \frac{9}{10} s_{\star}$, then $|\tilde{s}| \geq \frac{s_{\star}}{10}$. Hence, (23), the fact that $\varepsilon \leq \omega_{3}$, and $|\sigma(\tilde{y})| \leq 1$ give

$$
\begin{aligned}
\dot{V}_{2} & \leq-\frac{D_{\star}}{4 s} \tilde{s}^{2}-\frac{D_{\star}}{400 s} s_{\star}^{2}-\frac{s_{\star}}{2}\left[\frac{1}{L_{1}}-\frac{1}{L_{2}}\right] \varepsilon \tilde{y} \sigma(\tilde{y})-\tilde{Z}^{2}+\left\{\frac{\left[(a-1) s_{\text {in }}+s_{\star}^{2}\left(\frac{a}{L_{1}}-\frac{1}{L_{2}}\right)\right]^{2} \varepsilon^{2}}{D_{\star} s}\right\} \\
& \leq-\frac{D_{\star}}{4 s} \tilde{s}^{2}-\frac{s_{\star}}{2}\left[\frac{1}{L_{1}}-\frac{1}{L_{2}}\right] \varepsilon \tilde{y} \sigma(\tilde{y})-\tilde{Z}^{2}
\end{aligned}
$$

because $\varepsilon \leq \omega_{3}$ guarantees that the term in braces in (25) is at most $\frac{D_{\star}}{400 s} s_{\star}^{2}$. (Notice our use of $\frac{1}{20}$ in the formula for $\omega_{3}$.) In either case, we get (19). However, $V_{2}$ becomes unbounded as $\tilde{s} \rightarrow-s_{\star}$ so extra care is needed to obtain $\beta \in \mathcal{K} \mathcal{L}$ from the statement of the theorem; see Appendix A.1 below. This proves Theorem 1.

\section{Proof of Theorem 2}

We fix $\mathbf{d} \in \mathcal{D}_{2}(\bar{\Delta})$ and let $\Delta$ denote its sup norm, so $\Delta \leq \bar{\Delta}$. We use the notation from the proof of Theorem 1. We take

$$
\begin{aligned}
& C_{3}=2 \kappa_{1}+2\left[2 C_{2} \kappa_{1}+2 \max \left\{\bar{\alpha}, \bar{\beta} x_{1 \star} / x_{2 \star}\right\}\right] s_{\text {in }}+8 C_{2} s_{\text {in }}^{2} \text { and } \\
& W_{4}\left(\tilde{s}, \tilde{\xi}_{1}, \tilde{\xi}_{2}\right)=\frac{D_{\star}}{20 s} \tilde{s}^{2}+C_{1} \tilde{y} \sigma(\tilde{y})+\frac{1}{2} \tilde{Z}^{2},
\end{aligned}
$$

where we used the relations (7). Rearranging terms in (9) and again using (7) gives

$$
\left\{\begin{array}{l}
\dot{\tilde{s}}=D(y)\left[s_{\text {in }}-s\right]-\mu_{1}(s) x_{1}-\mu_{2}(s) x_{2}+g_{1}(\tilde{s}, y, \mathbf{d}) \\
\dot{\tilde{\xi}}_{i}=\mu_{i}(s)-D(y)-\mathbf{d}_{2}, \quad i=1,2
\end{array}\right.
$$

with $g_{1}(\tilde{s}, y, d)=d_{2}\left(-\tilde{s}+x_{1 \star}+x_{2 \star}\right)+\left[D(y)+d_{2}\right] d_{1}$. Along the trajectories of (27),

$$
\dot{V}_{2} \leq-W_{2}+\frac{\partial V_{2}}{\partial \tilde{s}} g_{1}(\tilde{s}, y, \mathbf{d})-\mathbf{d}_{2}\left[\frac{\partial V_{2}}{\partial \tilde{\xi}_{1}}+\frac{\partial V_{2}}{\partial \tilde{\xi}_{2}}\right]
$$


by (19). In terms of the constants from (11) and (7), we have $\left|g_{1}(\tilde{s}, y, \mathbf{d})\right| \leq \Delta\left(\kappa_{1}+|\tilde{s}|\right)$,

$$
\begin{aligned}
& W_{2}\left(\tilde{s}, \tilde{\xi}_{1}, \tilde{\xi}_{2}\right)=\frac{D_{\star}}{4 s} \tilde{s}^{2}+C_{1} \tilde{y} \sigma(\tilde{y})+\tilde{Z}^{2}, \\
& \frac{\partial V_{2}}{\partial \tilde{s}}=\frac{\tilde{s}}{s}+C_{2} \tilde{Z}, \quad \frac{\partial V_{2}}{\partial \tilde{\xi}_{1}}=\bar{\alpha} \tilde{x}_{1}+C_{2} \tilde{Z} x_{1}, \text { and } \frac{\partial V_{2}}{\partial \tilde{\xi}_{2}}=\frac{x_{1 \star}}{x_{2 \star}} \bar{\beta} \tilde{x}_{2}+C_{2} \tilde{Z} x_{2},
\end{aligned}
$$

where we used $\bar{\Delta} \leq \min \left\{1, D_{\star} / 5\right\}$ (which holds regardless of the choice of $\kappa_{1}>0$, by (12)) to get the bound on $\left|g_{1}(\tilde{s}, y, d)\right|$. Set $\tilde{v}=|\tilde{s}|+\left|\tilde{x}_{1}\right|+\left|\tilde{x}_{2}\right|$. From (28), we get

$$
\begin{aligned}
\dot{V}_{2} & \leq-W_{2}+\Delta \frac{\tilde{s}^{2}}{s}+\kappa_{1} \Delta \frac{|\tilde{s}|}{s}+\Delta \frac{x_{1 \star}}{x_{2 \star}}\left|\tilde{x}_{2}\right|+\Delta C_{2}|\tilde{Z}|\left(x_{1}+x_{2}+\kappa_{1}+|\tilde{s}|\right)+\Delta \bar{\alpha}\left|\tilde{x}_{1}\right| \\
& \leq-W_{2}+\Delta \frac{\tilde{s}^{2}}{s}+\kappa_{1} \Delta \frac{|\tilde{s}|}{s}+\Delta \frac{x_{1 \star}}{x_{2 \star}} \bar{\beta}\left|\tilde{x}_{2}\right|+\Delta C_{2}|\tilde{Z}|\left(2 \kappa_{1}+\tilde{v}\right)+\Delta \bar{\alpha}\left|\tilde{x}_{1}\right|,
\end{aligned}
$$

where we used $x_{1}+x_{2}+|\tilde{s}| \leq \tilde{v}+x_{1 \star}+x_{2 \star} \leq \tilde{v}+\kappa_{1}$. One easily shows that:

Lemma 1: For all $s>0$, we have $\frac{|\tilde{s}|}{s} \leq 2+\frac{3 \tilde{s}^{2}}{2 s_{\star} s}$.

See Appendix A.2 below for the proof. Combined with (30), Lemma 1 gives

$$
\dot{V}_{2} \leq-W_{2}\left(\tilde{s}, \tilde{\xi}_{1}, \tilde{\xi}_{2}\right)+\Delta\left(1+\frac{3 \kappa_{1}}{2 s_{\star}}\right) \frac{\tilde{s}^{2}}{s}+2 \kappa_{1} \Delta+\Delta C_{2}|\tilde{Z}|\left(2 \kappa_{1}+\tilde{v}\right)+\Delta \bar{\alpha}\left|\tilde{x}_{1}\right|+\Delta \frac{x_{1 \star}}{x_{2 \star}} \bar{\beta}\left|\tilde{x}_{2}\right| .
$$

From our choice of $\bar{\Delta}$ in (12) and the inequality $\Delta \leq \bar{\Delta}$, we get $\Delta\left(1+\frac{3 \kappa_{1}}{2 s_{\star}}\right) \leq \frac{D_{\star}}{5}$. Hence, the formula for $W_{2}$ given in (29) and $\tilde{Z}=\tilde{s}+\tilde{x}_{1}+\tilde{x}_{2}$ give

$$
\dot{V}_{2} \leq-W_{3}\left(\tilde{s}, \tilde{\xi}_{1}, \tilde{\xi}_{2}\right)+2 \kappa_{1} \Delta+\Delta C_{2}\left|\tilde{s}+\tilde{x}_{1}+\tilde{x}_{2}\right|\left(2 \kappa_{1}+\tilde{v}\right)+\Delta \bar{\alpha}\left|\tilde{x}_{1}\right|+\Delta \frac{x_{1 \star}}{x_{2 \star}} \bar{\beta}\left|\tilde{x}_{2}\right|
$$

where $W_{3}\left(\tilde{s}, \tilde{\xi}_{1}, \tilde{\xi}_{2}\right)=\frac{D_{\star}}{20 s} \tilde{s}^{2}+C_{1} \tilde{y} \sigma(\tilde{y})+\tilde{Z}^{2}$. We consider two cases:

lst case: $|\tilde{Z}| \geq 2 s_{\text {in. }}$. In Appendix A.2 below, we prove:

Lemma 2: If $|\tilde{Z}| \geq 2 s_{\text {in }}$, then $|\tilde{s}|+\left|\tilde{x}_{1}\right|+\left|\tilde{x}_{2}\right| \leq 2|\tilde{Z}|$.

We deduce that $\Delta \kappa_{1} \leq \Delta \kappa_{1}\left(\tilde{s}+\tilde{x}_{1}+\tilde{x}_{2}\right)^{2} /\left\{4 s_{\text {in }}^{2}\right\}, \Delta C_{2}\left[2 \kappa_{1}+\tilde{v}\right] \leq \Delta C_{2}\left[\frac{\kappa_{1}}{s_{\text {in }}}+2\right]|\tilde{Z}|$, and $\Delta\left\{\bar{\alpha}\left|\tilde{x}_{1}\right|+\bar{\beta}\left\{x_{1 \star} / x_{2 \star}\right\}\left|\tilde{x}_{2}\right|\right\} \leq \Delta \max \left\{\bar{\alpha}, \bar{\beta}\left\{x_{1 \star} / x_{2 \star}\right\}\right\} \tilde{Z}^{2} / s_{\text {in }}$. Substituting into (31) gives

$$
\begin{aligned}
\dot{V}_{2} \leq & -W_{3}\left(\tilde{s}, \tilde{\xi}_{1}, \tilde{\xi}_{2}\right)+\frac{\Delta \kappa_{1}}{2 s_{\text {in }}^{2}}\left(\tilde{s}+\tilde{x}_{1}+\tilde{x}_{2}\right)^{2}+\Delta C_{2}\left[\frac{\kappa_{1}}{s_{\text {in }}}+2\right]\left(\tilde{s}+\tilde{x}_{1}+\tilde{x}_{2}\right)^{2} \\
& +\frac{\Delta}{s_{\text {in }}} \max \left\{\bar{\alpha}, \bar{\beta}\left\{x_{1 \star} / x_{2 \star}\right\}\right\}\left(\tilde{s}+\tilde{x}_{1}+\tilde{x}_{2}\right)^{2} \\
= & -W_{3}\left(\tilde{s}, \tilde{\xi}_{1}, \tilde{\xi}_{2}\right)+\Delta \kappa_{2}\left(\tilde{s}+\tilde{x}_{1}+\tilde{x}_{2}\right)^{2},
\end{aligned}
$$

where we used (11). Since $\bar{\Delta} \leq 1 /\left(2 \kappa_{2}\right)$, we get $\dot{V}_{2} \leq-W_{4}\left(\tilde{s}, \tilde{\xi}_{1}, \tilde{\xi}_{2}\right)$; see (26).

2nd case: $\left|\tilde{s}+\tilde{x}_{1}+\tilde{x}_{2}\right| \leq 2 s_{\text {in }}$. One easily verifies:

Lemma 3: If $\left|\tilde{s}+\tilde{x}_{1}+\tilde{x}_{2}\right| \leq 2 s_{\text {in }}$, then $|\tilde{s}|+\left|\tilde{x}_{1}\right|+\left|\tilde{x}_{2}\right| \leq 4 s_{\text {in }}$.

See Appendix A.2 below for the proof. Using Lemma 3 and (31), we deduce in the second case that $\dot{V}_{2} \leq-W_{3}\left(\tilde{s}, \tilde{\xi}_{1}, \tilde{\xi}_{2}\right)+C_{3} \Delta \leq-W_{4}\left(\tilde{s}, \tilde{\xi}_{1}, \tilde{\xi}_{2}\right)+C_{3} \Delta$, so

$$
\dot{V}_{2} \leq-W_{4}\left(\tilde{s}, \tilde{\xi}_{1}, \tilde{\xi}_{2}\right)+C_{3} \Delta
$$


holds in both cases. Hence, if we define $\mathcal{V}$ and the positive definite function $\mathcal{W}$ by

$$
\mathcal{V}\left(\tilde{\Sigma}, \tilde{\xi}_{1}, \tilde{\xi}_{2}\right)=V_{2}\left(\left[e^{\tilde{\Sigma}}-1\right] s_{\star}, \tilde{\xi}_{1}, \tilde{\xi}_{2}\right) \quad \text { and } \quad \mathcal{W}\left(\tilde{\Sigma}, \tilde{\xi}_{1}, \tilde{\xi}_{2}\right)=W_{4}\left(\left[e^{\tilde{\Sigma}}-1\right] s_{\star}, \tilde{\xi}_{1}, \tilde{\xi}_{2}\right)
$$

we get $\dot{\mathcal{V}} \leq-\mathcal{W}+C_{3} \Delta$. We can also find $\alpha_{1}, \alpha_{2} \in \mathcal{K}_{\infty}$ so that $\alpha_{1}\left(\left|\left(\tilde{\Sigma}, \tilde{\xi}_{1}, \tilde{\xi}_{2}\right)\right|\right) \leq \mathcal{V}\left(\tilde{\Sigma}, \tilde{\xi}_{1}, \tilde{\xi}_{2}\right) \leq$ $\alpha_{2}\left(\left|\left(\tilde{\Sigma}, \tilde{\xi}_{1}, \tilde{\xi}_{2}\right)\right|\right)$; see Appendix A.1 below. Hence, a standard argument [1, p.1088] gives our estimate (iISS) with $\alpha=\alpha_{1} /\left(2 C_{3}\right)$, proving Theorem 2.

Remark 3: The system (9) is ISS in $\left(\tilde{\Sigma}, \tilde{\xi}_{1}, \tilde{\xi}_{2}\right)$ when $\mathbf{d} \in \mathcal{D}_{2}\left(\Delta^{b}\right)$, where, in terms of (12),

$$
\bar{\Delta}^{b}=\min \left\{\bar{\Delta}, \frac{1}{2 C_{3}} \min _{i} \tilde{\alpha}_{1}\left(x_{i \star} / 2\right)\right\}
$$

$\tilde{\alpha}_{1} \in \mathcal{K}_{\infty}$ is chosen so that $\tilde{\alpha}_{1}(r) \leq r^{2}$ and $W_{4}\left(\tilde{s}, \tilde{\xi}_{1}, \tilde{\xi}_{2}\right) \geq \tilde{\alpha}_{1}\left(\left|\left(\tilde{s}, \tilde{x}_{1}, \tilde{x}_{2}\right)\right|\right)$ hold everywhere; $\tilde{x}_{i}$ and $\tilde{\xi}_{i}$ are again related by (7); and $C_{3}$ is from (26). For the proof, see Appendix A.5 below.

Remark 4: An important special case of Theorem 2 is where only the dilution rate is affected by noise; i.e., $\mathbf{d}_{1}=0$ and $\mathbf{d}_{2} \neq 0$. This often occurs when the speed of the pump that supplies the chemostat with fresh nutrient is subjected to small fluctuations [10]. When $\mathbf{d}_{1}=0$, our proof of Theorem 2 remains valid if $\kappa_{1}$ is replaced by $x_{1 \star}+x_{2 \star}$. Another important case is where the noise term is only on the input nutrient concentration $s_{\text {in }}$, i.e., $\mathbf{d}_{2}=0$ and $\mathbf{d}_{1} \neq 0$, in which case Theorem 2 remains true if $\bar{\Delta}$ is replaced by

$$
\bar{\Delta}=\frac{0.16 D_{\star} s_{\star}}{D_{\star}+\varepsilon|a-1|} ;
$$

see Appendix A.3 below for the proof. We illustrate these special cases below.

Remark 5: Our bound (12) can be enlarged by reducing $\kappa_{2}$. For example, arguing as in the proof of Theorem 2 except separately considering the cases $\left|\tilde{s}+\tilde{x}_{1}+\tilde{x}_{2}\right| \geq M s_{\text {in }}$ and $\mid \tilde{s}+\tilde{x}_{1}+$ $\tilde{x}_{2} \mid \leq M s_{\text {in }}$ (for any constant $M \geq 2$ ) shows that Theorem 2 still holds if $\kappa_{2}$ is replaced by

$$
\kappa_{2}=\frac{2 \kappa_{1}}{M^{2} s_{\mathrm{in}}^{2}}+2 C_{2}+\frac{1}{M s_{\mathrm{in}}}\left[2 \kappa_{1} C_{2}+2 \bar{\alpha}+\frac{2 x_{1 \star}}{x_{2 \star}} \bar{\beta}\right]
$$

so $\kappa_{2} \approx 2 C_{2}$ for large $M$, if $C_{3}$ is appropriately enlarged. For details, see Appendix A.6 below.

\section{EXAMPLE}

We use the following special case of (4)-(5):

$$
\left\{\begin{aligned}
\dot{s} & =\left(D(y)+\mathbf{d}_{2}\right)\left(s_{\mathrm{in}}+\mathbf{d}_{1}-s\right)-\frac{0.05 s x_{1}}{20+s}-\frac{.052 s x_{2}}{25+s} \\
\dot{x}_{1} & =\left[\frac{.05 s}{20+s}-D(y)-\mathbf{d}_{2}\right] x_{1}, \quad \dot{x}_{2}=\left[\frac{.052 s}{25+s}-D(y)-\mathbf{d}_{2}\right] x_{2}
\end{aligned}\right.
$$

with $y=x_{1}+0.8 x_{2}$. We take $x_{1 \star}=0.05$ and $x_{2 \star}=0.02$. Using the notation from Theorem $1, a=$ $0.8, s_{\star}=105$, and $\varepsilon \in(0, .00753]$. We take $s_{\text {in }}=105.07$ and $D(y)=.042+0.001506 \sigma(y-0.066)$ 
where $\sigma(r)=\frac{r}{\sqrt{1+r^{2}}}$. Theorem 1 implies that regardless of the initial values, all trajectories of (36) with our controllers $D$ and $s_{\text {in }}$ converge to $(105,0.05,0.02)$ when $\mathbf{d}=0$.

We next consider the important special case where $\mathbf{d}_{1}=0$ but $\mathbf{d}_{2} \neq 0$. In that case, we can take $\kappa_{1}=x_{1 \star}+x_{2 \star}$; see Remark 4. Taking $\bar{\Delta}$ as in (12) and $\kappa_{2}$ as in Remark 5 with $M=25$ gives $\bar{\Delta} \approx 0.20 D_{\star}$. We chose $M=25$ to produce a larger disturbance threshold than the one we would get with a smaller $M$. By Theorem 2, the stability has the highly desirable property of robustness (in the iISS sense) to the noise $\mathbf{d}=\left(0, \mathbf{d}_{2}\right)$, provided $\left|\mathbf{d}_{2}\right|_{\infty}$ is no more than (about) $20 \%$ of $D$; in particular, if $\mathbf{d}_{2}$ is also integrable, then we have persistence of both species.

If instead the disturbance is only added to $s_{\mathrm{in}}$, then we can use (35) to get robustness to disturbances $\mathbf{d}_{1}$ that are bounded by $\bar{\Delta} \approx 16$, or about $15 \%$ of $s_{\text {in }}=105.07$. We simulated the corresponding dynamics (36) with the realistic sinusoidal input nutrient concentration disturbance $\mathbf{d}(t)=(0,0.01 \sin (t))$ and the initial value $\left(s, x_{1}, x_{2}\right)(0)=(103,2,1)$ and obtained the figures below with $\mathrm{g} / \mathrm{l}$ plotted against time in hours. This illustrates the persistence of $\left(x_{1}, x_{2}\right)(t)$ and the convergence $\left(s(t), x_{1}(t), x_{2}(t)\right) \rightarrow(105,0.05,0.02)$, with an overshoot determined by the iISS estimate and the magnitude of $\mathbf{d}_{1}$, and so validates our theorems.
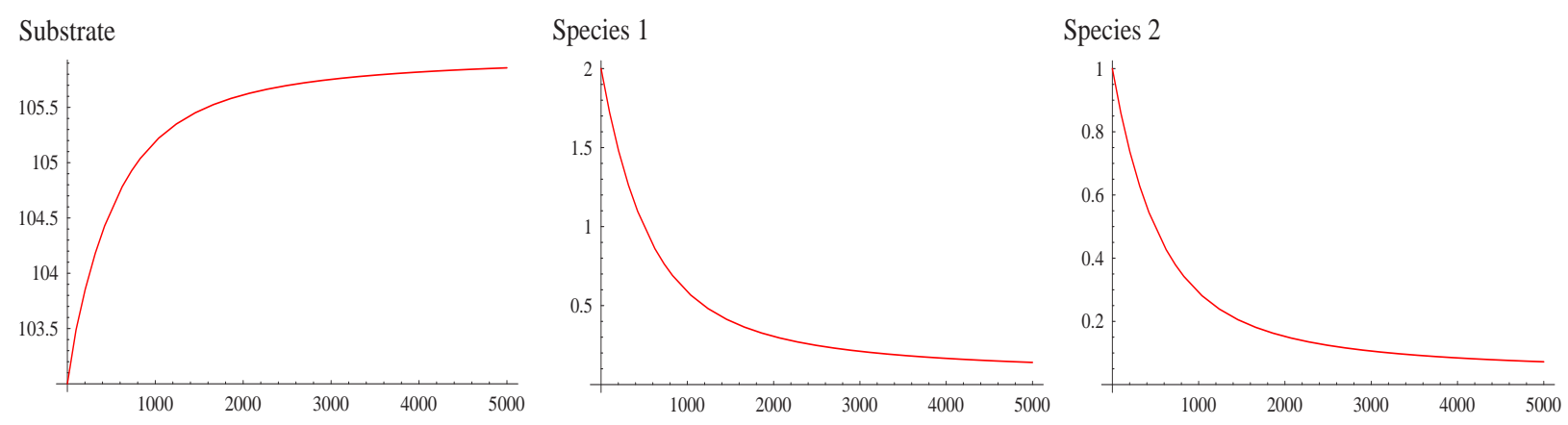

\section{APPENDICES}

\section{A.1. Stability Estimate From Theorem 1}

We provide the variant of the standard argument needed to obtain $\beta \in \mathcal{K} \mathcal{L}$ from Theorem 1. This will give the desired stability estimate in the transformed error vector $\left(\tilde{\Sigma}, \tilde{\xi}_{1}, \tilde{\xi}_{2}\right)$. We use the notation from the proof of Theorem 1. Using formulas (10), (14), and (18), one checks that

$$
\begin{aligned}
V_{2}\left(\tilde{s}, \tilde{\xi}_{1}, \tilde{\xi}_{2}\right) & \geq\left\{\frac{s_{\star}}{x_{1 \star}}\left(e^{\tilde{\Sigma}}-1-\tilde{\Sigma}\right)+\bar{\alpha}\left(e^{\tilde{\xi}_{1}}-1-\tilde{\xi}_{1}\right)+\bar{\beta}\left(e^{\tilde{\xi}_{2}}-1-\tilde{\xi}_{2}\right)\right\} x_{1 \star} \\
& \geq x_{1 \star} \min \left\{\frac{s_{\star}}{x_{\star}}, \bar{\alpha}, \bar{\beta}\right\}\left[E(|\tilde{\Sigma}|)+E\left(\left|\tilde{\xi}_{1}\right|\right)+E\left(\left|\tilde{\xi}_{2}\right|\right)\right] \geq \alpha_{1}\left(\left|\left(\tilde{\Sigma}, \tilde{\xi}_{1}, \tilde{\xi}_{2}\right)\right|\right),
\end{aligned}
$$


where $E(p)=p-1+e^{-p} ; \bar{\alpha}$ and $\bar{\beta}$ are defined in (10); and $\alpha_{1}(r)=x_{1 \star} \min \left\{\frac{s_{\star}}{x_{1 \star}}, \bar{\alpha}, \bar{\beta}\right\} E(r / 3)$. (We used the general relation $E(p+q+r) \leq E(3 p)+E(3 q)+E(3 r)$ for all $p, q, r \geq 0$, and the fact that $e^{r}-1-r \geq e^{-r}-1+r$ for all $r \geq 0$.) Since $E \in \mathcal{K}_{\infty}$, we get $\alpha_{1} \in \mathcal{K}_{\infty}$. We can also find $\alpha_{2} \in$ $\mathcal{K}_{\infty}$ so that $V_{2}\left(\tilde{s}, \tilde{\xi}_{1}, \tilde{\xi}_{2}\right) \leq \alpha_{2}\left(\left|\left(\tilde{\Sigma}, \tilde{\xi}_{1}, \tilde{\xi}_{2}\right)\right|\right)$, everywhere, e.g., $\alpha_{2}(r)=\bar{K}_{1} E(-r)+\bar{K}_{2}\left(e^{r}-1\right)^{2}$ for large enough constants $\bar{K}_{1}, \bar{K}_{2}>0$ (since $r \mapsto E(-r)$ is also of class $\mathcal{K}_{\infty}$ ). Defining $\mathcal{V}$ and $\mathcal{W}$ as in (33) except with $W_{2}$ instead of $W_{4}$ gives $\alpha_{1}\left(\left|\left(\tilde{\Sigma}, \tilde{\xi}_{1}, \tilde{\xi}_{2}\right)\right|\right) \leq \mathcal{V}\left(\tilde{\Sigma}, \tilde{\xi}_{1}, \tilde{\xi}_{2}\right) \leq \alpha_{2}\left(\left|\left(\tilde{\Sigma}, \tilde{\xi}_{1}, \tilde{\xi}_{2}\right)\right|\right)$ everywhere. Also, $\dot{\mathcal{V}} \leq-\mathcal{W}$ everywhere, by (19). Since $\mathcal{W}$ is positive definite, the desired estimate then follows from the standard argument, e.g. [1] with controls $\mu=0$ and the positive definite function $\alpha_{3}(r)=\min \left\{\mathcal{W}\left(\tilde{\Sigma}, \tilde{\xi}_{1}, \tilde{\xi}_{2}\right):\left|\left(\tilde{\Sigma}, \tilde{\xi}_{1}, \tilde{\xi}_{2}\right)\right|=r\right\}$.

\section{A.2. Proofs of Lemmas 1-3}

To prove Lemma 1 , we consider the following cases. If $\tilde{s} \geq 0$, then $|\tilde{s}| / s=\tilde{s} / s \leq 1$. Assume next that $\tilde{s} \leq 0$ and $s \geq s_{\star} / 3$. Then $|\tilde{s}|=-\tilde{s}=-s+s_{\star} \leq 2 s$, so $|\tilde{s}| / s \leq 2$. Finally, assume that $\tilde{s} \leq 0$ and $s \leq s_{\star} / 3$. Then $|\tilde{s}|=-\tilde{s} \geq 2 s_{\star} / 3$, so $\frac{3}{2} \tilde{s}^{2} /\left(s s_{\star}\right) \geq|\tilde{s}| / s$. This proves the lemma.

To prove Lemma 2, first notice that $\left|\tilde{s}+\tilde{x}_{1}+\tilde{x}_{2}\right| \leq|\tilde{s}|+\left|\tilde{x}_{1}\right|+\left|\tilde{x}_{2}\right| \leq s+x_{1}+x_{2}+s_{\text {in }}=$ $\tilde{s}+\tilde{x}_{1}+\tilde{x}_{2}+2 s_{\text {in }}$. Hence, if $\left|\tilde{s}+\tilde{x}_{1}+\tilde{x}_{2}\right| \geq 2 s_{\text {in }}$, then $\tilde{s}+\tilde{x}_{1}+\tilde{x}_{2} \geq 0$, so $\tilde{s}+\tilde{x}_{1}+\tilde{x}_{2} \geq 2 s_{\text {in }}$. Therefore, $|\tilde{s}|+\left|\tilde{x}_{1}\right|+\left|\tilde{x}_{2}\right| \leq 2\left(\tilde{s}+\tilde{x}_{1}+\tilde{x}_{2}\right)=2\left|\tilde{s}+\tilde{x}_{1}+\tilde{x}_{2}\right|$, proving the lemma.

To prove Lemma 3, notice that if $\left|\tilde{s}+\tilde{x}_{1}+\tilde{x}_{2}\right| \leq 2 s_{\text {in }}$, then $|\tilde{s}|+\left|\tilde{x}_{1}\right|+\left|\tilde{x}_{2}\right| \leq s+x_{1}+x_{2}+s_{\text {in }} \leq$ $s+x_{1}+x_{2}-s_{\text {in }}+2 s_{\text {in }}=\tilde{s}+\tilde{x}_{1}+\tilde{x}_{2}+2 s_{\text {in }} \leq\left|\tilde{s}+\tilde{x}_{1}+\tilde{x}_{2}\right|+2 s_{\text {in }} \leq 4 s_{\text {in }}$.

\section{A.3. Disturbances Only On $\mathrm{s}_{\text {in }}$}

We prove the assertion from Remark 4 that in the special case where the disturbance is only on the input nutrient concentration $s_{\text {in }}$ (i.e., $\mathbf{d}_{1} \neq 0$ and $\mathbf{d}_{2}=0$ ), the disturbance bound in Theorem 2 can instead be taken to be (35). We indicate the changes needed in the proof of Theorem 2. Choose $\bar{\Delta}$ as in (35). Since $\mathbf{d}_{2}=0,\left|g_{1}(\tilde{s}, y, \mathbf{d})\right| \leq\left(D_{*}+\varepsilon|a-1|\right) \Delta$, so instead of (30),

$$
\begin{aligned}
\dot{V}_{2} & \leq-W_{2}+\Delta\left\{\frac{|\tilde{s}|}{s}+C_{2}|\tilde{Z}|\right\}\left(D_{\star}+\varepsilon|a-1|\right) \\
& \leq-W_{2}+\frac{1}{2} \tilde{Z}^{2}+\Delta \frac{|\tilde{s}|}{s}\left(D_{\star}+\varepsilon|a-1|\right)+M \Delta .
\end{aligned}
$$

where $M=\frac{\bar{\Delta}}{2} C_{2}^{2}\left(D_{\star}+\varepsilon|a-1|\right)^{2}$ and the last inequality used $\Delta \leq \bar{\Delta}$ and the relation $\operatorname{lm} \leq$ $\frac{1}{2} l^{2}+\frac{1}{2} m^{2}$. Consider two cases. If $|\tilde{s}| \geq 4 s_{\star} / 5$, then $\left(5|\tilde{s}| /\left\{4 s_{\star}\right\}\right)^{2} \geq 1$ gives

$$
\begin{aligned}
\dot{V}_{2} & \leq-W_{2}+\frac{1}{2} \tilde{Z}^{2}+\Delta \frac{1}{s}\left\{\frac{5|\tilde{s}| /\left\{4 s_{\star}\right\}}{5 /\left\{4 s_{\star}\right\}}\right\}\left(D_{\star}+\varepsilon|a-1|\right)+M \Delta \\
& \leq-W_{2}+\frac{1}{2} \tilde{Z}^{2}+\Delta \frac{5}{4 s_{\star}} \frac{\tilde{s}^{2}}{s}\left(D_{\star}+\varepsilon|a-1|\right)+M \Delta \leq-W_{4}+M \Delta,
\end{aligned}
$$


where $W_{4}$ is from (26), since $\Delta \leq \bar{\Delta}$ as given by (35). On the other hand, if $|\tilde{s}| \leq 4 s_{\star} / 5$, then $s \geq s_{\star} / 5$, hence $|\tilde{s}| / s \leq 4$. This and (A.1) give $\dot{V}_{2} \leq-W_{4}+M_{1} \Delta$ in either case, where $M_{1}=M+4\left(D_{\star}+\varepsilon|a-1|\right)$. The argument concludes exactly as in the last part of the proof of Theorem 2, except with $C_{3}$ replaced by $M_{1}$.

\section{A.4. LOWER BOUND FOR $W_{4}$}

We construct a function $\tilde{\alpha}_{1} \in \mathcal{K}_{\infty}$ so that $W_{4}\left(\tilde{s}, \tilde{\xi}_{1}, \tilde{\xi}_{2}\right) \geq \tilde{\alpha}_{1}\left(\left|\left(\tilde{s}, \tilde{x}_{1}, \tilde{x}_{2}\right)\right|\right)$ and $\tilde{\alpha}_{1}(r) \leq r^{2}$ everywhere, where $\tilde{x}_{i}$ and $\tilde{\xi}_{i}$ are related as in (7) as before and $W_{4}$ is defined in (26), as required by Remark 3. Set $N=\left|\left(\tilde{s}, \tilde{x}_{1}, \tilde{x}_{2}\right)\right|$. Then $s=s_{\star}+\tilde{s} \leq s_{\star}+N$ and $\sqrt{1+\left(\tilde{x}_{1}+a \tilde{x}_{2}\right)^{2}} \leq$ $\sqrt{2}+\sqrt{2}(a+1) N$ hold everywhere. It follows from our choice of $\sigma$ that $W_{4}\left(\tilde{s}, \tilde{\xi}_{1}, \tilde{\xi}_{2}\right) \geq$ $K(N)\left[\tilde{s}^{2}+\left(\tilde{x}_{1}+a \tilde{x}_{2}\right)^{2}+\left(\tilde{s}+\tilde{x}_{1}+\tilde{x}_{2}\right)^{2}\right]$ everywhere, where $K(N)=\min \left\{\frac{D_{\star}}{20}, C_{1}, \frac{1}{2}\right\} /\left\{s_{\star}+\right.$ $\sqrt{2}\{1+(a+1) N\}\}$. Since $a \neq 1, Q\left(\tilde{s}, \tilde{x}_{1}, \tilde{x}_{2}\right)=\tilde{s}^{2}+\left(\tilde{x}_{1}+a \tilde{x}_{2}\right)^{2}+\left(\tilde{s}+\tilde{x}_{1}+\tilde{x}_{2}\right)^{2}$ is positive definite. Let $\lambda_{\min }>0$ be the smallest eigenvalue of the positive definite matrix $P$ for which $Q\left(\tilde{s}, \tilde{x}_{1}, \tilde{x}_{2}\right)=\left(\tilde{s}, \tilde{x}_{1}, \tilde{x}_{2}\right)^{T} P\left(\tilde{s}, \tilde{x}_{1}, \tilde{x}_{2}\right)$. Since $Q\left(\tilde{s}, \tilde{x}_{1}, \tilde{x}_{2}\right) \geq N^{2} \lambda_{\min }$ everywhere, it follows that $\tilde{\alpha}_{1}(N)=\min \left\{1, \lambda_{\min }\right\} K(N) N^{2}$ satisfies the requirements. Moreover, $\tilde{\alpha}_{1}(r) \leq r^{2}$ everywhere.

\section{A.5. Disturbance BoUnd FOR ISS}

We prove the ISS assertion from Remark 3, using the function $\tilde{\alpha}_{1} \in \mathcal{K}_{\infty}$ from Appendix A.4. We indicate the changes necessary in the proof of Theorem 2. Arguing as in the proof of Theorem 2 with $\Delta:=|\mathbf{d}|_{\infty} \leq \bar{\Delta}$ yields (32) along all trajectories of the error dynamics. Set

$$
\bar{s}=\min \left\{\frac{s_{\star}}{2}, \frac{D_{\star} s_{\star}^{2}}{80\left[C_{3} \bar{\Delta}+2 s_{\mathrm{in}}^{2}\right]}\right\} .
$$

We next transform (32) into an ISS decay estimate in the transformed error vector $\left(\tilde{\Sigma}, \tilde{\xi}_{1}, \tilde{\xi}_{2}\right)$ from (7). To do so, we view (32) simply as a relation in $\tilde{s}, \tilde{\xi}_{1}, \tilde{\xi}_{2}$, and $\Delta \in\left[0, \bar{\Delta}^{b}\right]$, rather than an estimate along trajectories. Here $\bar{\Delta}^{b}$ is the tighter ISS bound in (34). We consider two cases.

Case I. Assume that $\left|\tilde{s}+\tilde{x}_{1}+\tilde{x}_{2}\right| \geq 2 s_{\text {in }}$ or $s \leq \bar{s}$ or $\left|\tilde{x}_{1}\right| \geq x_{1 \star} / 2$ or $\left|\tilde{x}_{2}\right| \geq x_{2 \star} / 2$. If $|\tilde{Z}| \geq 2 s_{\text {in }}$, then $W_{4}\left(\tilde{s}, \tilde{\xi}_{1}, \tilde{\xi}_{2}\right) \geq 2 s_{\text {in }}^{2}$, so since $\bar{\Delta}^{b} \leq s_{\text {in }}^{2} / C_{3}$ (which follows because $\tilde{\alpha}_{1}(r) \leq r^{2}$ ), we also have $\dot{V}_{2} \leq-2 s_{\text {in }}^{2}+C_{3} \Delta \leq-s_{\text {in }}^{2}$. If $s \leq \bar{s}$, then $|\tilde{s}| \geq \frac{s_{\star}}{2}$, so (32) and (A.2) give $\dot{V}_{2} \leq-D_{\star} \tilde{s}^{2} /(20 \bar{s})+C_{3} \Delta \leq-2 s_{\text {in }}^{2}$. Since $\bar{\Delta}^{b} \leq \frac{1}{2 C_{3}} \min _{i} \tilde{\alpha}_{1}\left(x_{i \star} / 2\right)$, condition (32) and our choice of $\tilde{\alpha}_{1}$ give: If either $\left|\tilde{x}_{1}\right| \geq x_{1 \star} / 2$ or $\left|\tilde{x}_{2}\right| \geq x_{2 \star} / 2$, then $\dot{V}_{2} \leq-\min _{i} \tilde{\alpha}_{1}\left(x_{i \star} / 2\right)+C_{3} \Delta \leq$ $-\frac{1}{2} \min _{i} \tilde{\alpha}_{1}\left(x_{i \star} / 2\right)$. Hence, $\dot{V}_{2} \leq-\min \left\{s_{\text {in }}^{2}, \frac{1}{2} \min _{i} \tilde{\alpha}_{1}\left(x_{i \star} / 2\right)\right\}$ if Case I occurs. 
Case II. Assume that $\left|\tilde{s}+\tilde{x}_{1}+\tilde{x}_{2}\right| \leq 2 s_{\text {in }}, s \geq \bar{s},\left|\tilde{x}_{1}\right| \leq x_{1 \star} / 2$ and $\left|\tilde{x}_{2}\right| \leq x_{2 \star} / 2$. Since $\left|\tilde{x}_{1}\right| \leq x_{1 \star} / 2$ and $\left|\tilde{x}_{2}\right| \leq x_{2 \star} / 2$, we get $A_{i}\left(\tilde{\xi}_{i}\right) \leq\left(\tilde{x}_{i} / x_{i \star}\right)^{2}$ for $i=1,2$ (using (7), (10), and the fact that $\phi(p)=\ln (p+1)-p+p^{2}$ is nonnegative when $p \geq-1 / 2$ and then choosing $\left.p=\tilde{x}_{i} / x_{i \star}\right)$. Since $s \geq \bar{s}$, we can find a constant $c>0$ so that $A_{o}(\tilde{s}) \leq c \tilde{s}^{2}$. (To see why, first notice that if $s \geq s_{\star} / 2$, then $\tilde{s} / s_{\star} \geq-1 / 2$, in which case we can apply our remark on $\phi(p)$ to get

$$
A_{o}(\tilde{s})=s_{\star}\left[\frac{\tilde{s}}{s_{\star}}-\ln \left(1+\frac{\tilde{s}}{s_{\star}}\right)\right] \leq s_{\star}\left(\frac{\tilde{s}}{s_{\star}}\right)^{2} .
$$

On the other hand, if $\bar{s} \leq s \leq s_{\star} / 2$, then $A_{o}(\tilde{s})=\tilde{s}-s_{\star} \ln \left(s / s_{\star}\right)$ stays bounded and $\tilde{s} \leq-s_{\star} / 2$ implies $\tilde{s}^{2}$ is bounded away from zero, so $A_{o}(\tilde{s}) / \tilde{s}^{2}$ stays bounded.) This guarantees that there is a quadratic function $c_{1} \tilde{s}^{2}+c_{2} \tilde{x}_{1}^{2}+c_{3} \tilde{x}_{2}^{2}$ that is an upper bound for $V_{2}$ on the set where Case II holds. By Lemma $3, \max \left\{|\tilde{s}|,\left|\tilde{x}_{1}\right|,\left|\tilde{x}_{2}\right|\right\} \leq 4 s_{\text {in }}$, so we can easily find a constant $C_{b}>0$ such that in Case II, $V_{2}\left(\tilde{s}, \tilde{\xi}_{1}, \tilde{\xi}_{2}\right) \leq C_{b} \tilde{\alpha}_{1}\left(\left|\left(\tilde{s}, \tilde{x}_{1}, \tilde{x}_{2}\right)\right|\right)$. Again using Lemma 3 and (32), we can then find a constant $C_{d}>0$ so that $\dot{V}_{2} \leq-V_{2}\left(\tilde{s}, \tilde{\xi}_{1}, \tilde{\xi}_{2}\right) / C_{b}+C_{d} \Delta / e^{V_{2}\left(\tilde{s}_{,}, \tilde{\xi}_{1}, \tilde{\xi}_{2}\right)}$ if Case II occurs.

By separately considering the conclusions of Cases I-II and setting $V_{3}\left(\tilde{s}, \tilde{\xi}_{1}, \tilde{\xi}_{2}\right)=e^{V_{2}\left(\tilde{s}, \tilde{\xi}_{1}, \tilde{\xi}_{2}\right)}-1$, we immediately obtain $\dot{V}_{3} \leq-\min \left\{s_{\text {in }}^{2}, \frac{1}{2} \min _{i} \tilde{\alpha}_{1}\left(x_{i \star} / 2\right), \frac{1}{C_{b}}\right\} V_{3}+C_{d}|\mathbf{d}|_{\infty}$ along all trajectories of the error dynamics for which $|\mathbf{d}|_{\infty} \leq \Delta^{b}$, where we used the fact that $r e^{r} \geq e^{r}-1$ for all $r \geq 0$. Setting $\mathcal{M}(r)=e^{r}-1$, it follows that the function $J\left(\tilde{\Sigma}, \tilde{\xi}_{1}, \tilde{\xi}_{2}\right)=V_{3}\left(\left(e^{\tilde{\Sigma}}-1\right) s_{\star}, \tilde{\xi}_{1}, \tilde{\xi}_{2}\right)$

satisfies $\dot{J} \leq-\min \left\{s_{\text {in }}^{2}, \frac{1}{2} \min _{i} \tilde{\alpha}_{1}\left(x_{i \star} / 2\right), \frac{1}{C_{b}}\right\} J+C_{d}|\mathbf{d}|_{\infty}$ and $\underline{\alpha}_{J}\left(\left|\left(\tilde{\Sigma}, \tilde{\xi}_{1}, \tilde{\xi}_{2}\right)\right|\right) \leq J\left(\tilde{\Sigma}, \tilde{\xi}_{1}, \tilde{\xi}_{2}\right) \leq$ $\bar{\alpha}_{J}\left(\left|\left(\tilde{\Sigma}, \tilde{\xi}_{1}, \tilde{\xi}_{2}\right)\right|\right)$ along all trajectories for the error dynamics, where $\underline{\alpha}_{J}=\mathcal{M} \circ \alpha_{1}, \bar{\alpha}_{J}=\mathcal{M} \circ \alpha_{2}$, and $\alpha_{1}, \alpha_{2} \in \mathcal{K}_{\infty}$ are the lower and upper bounds for $V_{2}$ constructed in Appendix A.1 above, respectively. Hence, standard arguments yield the desired ISS estimate for suitable functions $\beta \in \mathcal{K} \mathcal{L}$ and $\gamma \in \mathcal{K}_{\infty}$ [14]. Thus, (9) is ISS in the variable $\left(\tilde{\Sigma}, \tilde{\xi}_{1}, \tilde{\xi}_{2}\right)$ with respect to the disturbance $\mathbf{d}:[0, \infty) \rightarrow \bar{\Delta}^{b} \mathcal{B}_{2}$, as claimed.

\section{A.6. Conservativeness of the Disturbance Bound}

We prove the assertion from Remark 5, thus providing a method for enlarging the bound (12) for the disturbance $\mathbf{d}$. We indicate the changes needed in the proof of Theorem 2. We argue as in the earlier proof up through (31), and we let $M \geq 2$ be any constant. Consider two cases.

Case 1M: If $\left|\tilde{s}+\tilde{x}_{1}+\tilde{x}_{2}\right| \geq M s_{\text {in }}$, then $\tilde{v}=|\tilde{s}|+\left|\tilde{x}_{1}\right|+\left|\tilde{x}_{2}\right| \leq 2\left|\tilde{s}+\tilde{x}_{1}+\tilde{x}_{2}\right|$, by Lemma 2 (since $M \geq 2$ ). We deduce that $\Delta \kappa_{1} \leq \Delta \kappa_{1}\left(\tilde{s}+\tilde{x}_{1}+\tilde{x}_{2}\right)^{2} /\left\{M^{2} s_{\text {in }}^{2}\right\}$,

$$
\Delta C_{2}\left[2 \kappa_{1}+\tilde{v}\right] \leq \Delta C_{2}\left[\frac{2 \kappa_{1}}{M s_{\text {in }}}\left|\tilde{s}+\tilde{x}_{1}+\tilde{x}_{2}\right|+\tilde{v}\right] \leq \Delta C_{2}\left[\frac{2 \kappa_{1}}{M s_{\text {in }}}+2\right]\left|\tilde{s}+\tilde{x}_{1}+\tilde{x}_{2}\right|
$$


$\Delta \bar{\alpha}\left|\tilde{x}_{1}\right| \leq 2 \Delta \bar{\alpha}\left(\tilde{s}+\tilde{x}_{1}+\tilde{x}_{2}\right)^{2} /\left\{M s_{\text {in }}\right\}$, and $\Delta \bar{\beta}\left|\tilde{x}_{2}\right| \leq 2 \Delta \bar{\beta}\left(\tilde{s}+\tilde{x}_{1}+\tilde{x}_{2}\right)^{2} /\left\{M s_{\text {in }}\right\}$. Substituting into (31), recalling that $\Delta \leq 1 /\left\{2 \kappa_{2}\right\}$, and grouping terms now gives $V_{2} \leq-W_{3}\left(\tilde{s}_{3}, \tilde{\xi}_{1}, \tilde{\xi}_{2}\right)+\Delta \kappa_{2} \tilde{Z}^{2} \leq$ $-W_{4}\left(\tilde{s}, \tilde{\xi}_{1}, \tilde{\xi}_{2}\right)$ where now $\kappa_{2}$ is redefined as in Remark 5.

Case 2M: If $\left|\tilde{s}+\tilde{x}_{1}+\tilde{x}_{2}\right| \leq M s_{\text {in }}$, then a slight variant of the proof of Lemma 3 gives $|\tilde{s}|+\left|\tilde{x}_{1}\right|+\left|\tilde{x}_{2}\right| \leq(2+M) s_{\text {in }}$. Therefore, (31) gives $\dot{V}_{2} \leq-W_{4}\left(\tilde{s}_{1}, \tilde{\xi}_{1}, \tilde{\xi}_{2}\right)+C_{3} \Delta$ where $C_{3}$ is now redefined to be $C_{3}=2 \kappa_{1}+C_{2} M(2+M) s_{\text {in }}^{2}+\left[2 M C_{2} \kappa_{1}+\left\{\bar{\alpha}+\left(x_{1 \star} / x_{2 \star}\right) \bar{\beta}\right\}(2+M)\right] s_{\text {in }}$. The rest of the proof is exactly as before.

\section{REFERENCES}

[1] D. Angeli, E.D. Sontag, and Y. Wang, "A characterization of integral input to state stability," IEEE Trans. Automat. Control, vol. 45, no. 6, pp. 1082-1097, June 2000.

[2] O. Bernard and J.-L. Gouzé, "Nonlinear qualitative signal processing for biological systems: Application to the algal growth in bioreactors," Mathematical Biosciences, vol. 157, nos. 1-2, pp. 357-372, 1999.

[3] M. Chaves, "Input-to-state stability of rate-controlled biochemical networks," SIAM J. Control Optim., vol. 44, no. 2, pp. 704-727, 2005.

[4] P. De Leenheer and H.L. Smith, "Feedback control for chemostat models," J. Math. Biol., vol. 46, no. 1, pp. 48-70, 2003.

[5] J.-L. Gouzé and G. Robledo, "Feedback control for competition models with different removal rates in the chemostat," INRIA rapport de Recherche 5555, 2005.

[6] —_ " "Robust control for an uncertain chemostat model," International J. Robust Nonlinear Control, vol. 16, no. 3, pp. 133-155, 2006.

[7] F. Grognard, F. Mazenc, and A. Rapaport, "Polytopic Lyapunov functions for persistence analysis of competing species," Discrete and Continuous Dynamical Systems-Series B, vol. 8, no. 1, pp. 73-93, July 2007.

[8] S.B. Hsu, "Limiting behavior for competing species," SIAM J. Applied Mathematics, vol. 34, no. 4, pp. 760-763, 1978.

[9] B. Li, "Asymptotic behavior of the chemostat: General responses and different removal rates," SIAM J. Applied Mathematics, vol. 59, no. 2, pp. 411-422, 1999.

[10] F. Mazenc, M. Malisoff, and P. De Leenheer, "On the stability of periodic solutions in the perturbed chemostat," Mathematical Biosciences and Engineering, vol. 4, no. 2, pp. 319-338, 2007.

[11] F. Mazenc, M. Malisoff, and J. Harmand, "Further results on stabilization of periodic trajectories for a chemostat with two species," IEEE Transactions on Automatic Control, Volume 53, Special Issue on Systems Biology, pp. 66-74, 2008.

[12] H.L. Smith and P. Waltman, The Theory of the Chemostat. Cambridge, U.K.: Cambridge University Press, 1995.

[13] E.D. Sontag, "Smooth stabilization implies coprime factorization," IEEE Trans. Automat. Control, vol. 34, no. 4, pp. 435-443, April 1989.

[14] — _ "Input-to-state stability: Basic concepts and results," in Nonlinear and Optimal Control Theory, P. Nistri and G. Stefani, Eds. Berlin, Germany: Springer-Verlag, 2006, pp. 163-220.

[15] G. Wolkowicz and Z. Lu, "Global dynamics of a mathematical model of competition in the chemostat: general response functions and different death rates," SIAM J. Applied Mathematics, vol. 52, no. 1, pp. 222-233, 1992. 